: AMIERICANOS ivv Revista de Estudios Transfronterizos
Volumen XX, número 1 , enero-junio de 2020.

Recibido: 4 de octubre de 2019. Aprobado: 30 de marzo de 2020.

\title{
El rol de los gobiernos locales en las investigaciones migratorias en Argentina*
}

\section{The role of local governments in migration research in Argentina}

\author{
Julieta Nicolao** \\ Comisión de Investigaciones Científicas de la Provincia de Buenos Aires (CIC), Buenos \\ Aires, Argentina.
}

\begin{abstract}
Resumen
Este artículo se propone realizar una revisión bibliográfica sobre la evolución del campo de los estudios migratorios en Argentina, reconociendo la necesidad de avanzar en investigaciones sobre el papel que desempeñan los gobiernos locales en materia de formulación e implementación de políticas migratorias. Se argumenta que, si bien los municipios no siempre cuentan con los soportes normativos, las competencias suficientes o los recursos necesarios, en la práctica deben dar respuesta a distintos temas asociados a la inserción social de los migrantes y al reconocimiento efectivo de derechos. Esta situación, que los convierte en actores cada vez más importantes en la gestión migratoria contemporánea, reclama una comprensión más acabada del sector académico especializado. Además de identificar este vacío de conocimiento, el trabajo propone
\end{abstract}

* Este trabajo se ha desarrollado en el marco del proyecto "Migración regional, política migratoria y derechos sociales en el interior bonaerense" (03-JOVIN-10D SECAT-UNCPBA), financiado por medio del Programa de Fortalecimiento de la Ciencia y Tecnología en Universidades Nacionales de la Secretaría de Políticas Universitarias del Ministerio de Educación argentino.

** Licenciada en Relaciones Internacionales, Universidad Nacional del Centro de la Provincia de Buenos Aires (UNCPBA). Doctora en Ciencia Política, Universidad Nacional de San Martín, Argentina. Investigadora asistente de la Comisión de Investigaciones Científicas de la Provincia de Buenos Aires (CIC) y miembro de la planta estable del Centro de Estudios Interdisciplinarios en Problemáticas Internacionales y Locales (CEIPIL-UNICEN-CIC). Dirección: Pinto 399, Tandil, Prov. de Buenos Aires, Argentina. Correo electrónico: nicolao_j@yahoo.com.ar

Cómo citar este artículo: Nicolao, J. (2020). El rol de los gobiernos locales en las investigaciones migratorias en Argentina. Si Somos Americanos. Revista de Estudios Transfronterizos, 20(1), 152-175. doi: 10.4067/S0719-09482020000100152 
algunas directrices para abordar la temática en futuras publicaciones, y destaca la necesaria incorporación de disciplinas sociales como la ciencia política y otras afines al estudio del Estado y las políticas públicas, entendiendo que están llamadas a realizar importantes contribuciones en este campo de estudios.

Palabras clave: estudios migratorios, Argentina, gobiernos locales.

\begin{abstract}
The purpose of this article is to undertake a review of the literature on developments in the area of migratory studies in Argentina. It recognizes the need to make progress in research on the role played by local governments in the formulation and implementation of migration policies. It is argued that, although the municipalities do not always have regulatory support, sufficient powers or the necessary resources, the reality is that they must respond to different issues associated with the social insertion of migrants and the effective recognition of their rights. This situation, which makes the municipalities increasingly important stakeholders in contemporary migration management, demands a more comprehensive understanding by the specialized academic sector. In addition to identifying this knowledge gap, the paper proposes various guidelines to address this issue in future publications. Furthermore, it highlights the necessary incorporation of social disciplines such as political science, and others related to the study of the State and public policies, with the understanding that they are called upon to make important contributions in this field of study.
\end{abstract}

Keywords: migratory studies, Argentina, local governments.

\title{
Introducción
}

El estudio de las migraciones internacionales ha sido abonado desde múltiples disciplinas sociales desde fines del siglo XIX y comienzos del XX, asociado al interés por analizar el fenómeno internacional de la migración de masas a escala global. Sin embargo, a pesar de esta extensa tradición académica, y de la temprana intervención que el Estado ejerció sobre los flujos internacionales de población, la inmigración fue concebida durante décadas como un fenómeno socioeconómico al que no se le atribuyó significación política.

Argentina, país de amplia tradición inmigratoria, no escapó a esta tendencia global, ya que si bien cuenta con tempranos antecedentes de investigaciones migratorias, los estudios que atienden el vínculo "migraciones-Estado", empezaron a desarrollarse de forma sistemática recién en la segunda mitad de la década de 1980. Desde entonces, ha venido abonándose 
con múltiples aportes, y recién en el decenio de 2000 se observó una injerencia más concreta de disciplinas como la ciencia política, las relaciones internacionales y las ciencias jurídicas (Domenech y Pereira, 2017). Quizá por esta razón, aún existen aristas muy poco estudiadas, pero de creciente interés en la actualidad, como es el abordaje de la cuestión migratoria desde la perspectiva de los gobiernos locales.

Este artículo se propone realizar un recorrido sobre el avance de este campo de estudios en Argentina, reconociendo la necesaria preocupación por entender el papel de las administraciones locales en materia de formulación e implementación de políticas migratorias. Se asume al respecto que, si bien los municipios no siempre cuentan con los soportes normativos, las competencias suficientes o los recursos necesarios, se ven obligados a dar respuesta a distintos temas asociados a la inserción social de los migrantes y al reconocimiento efectivo de derechos, en tanto constituyen el nivel del Estado más próximo a la población y el territorio, y son los principales receptores de sus demandas.

ara cumplir con el objetivo planteado, el texto se estructura en tres secciones: la primera se ocupa de recuperar brevemente la tardía incorporación del papel del Estado en los estudios migratorios y el contexto en el que comenzó a introducirse la dimensión política en las investigaciones sobre el tema. La segunda se concentra en la evolución de la literatura científica producida en Argentina en este campo, reconociendo el papel de los gobiernos locales como área de vacancia en el ámbito de las políticas migratorias. La tercera y última intenta brindar algunas de las razones centrales sobre la relevancia de atender, reflexionar y comprender esta dimensión específica.

\section{Estado y migraciones internacionales. La tardía incorporación de la dimensión política en los estudios migratorios}

Los Estados-nación han tratado desde su misma creación de monopolizar los legítimos medios de movimiento de personas, concentrando en sus manos el derecho exclusivo para autorizar y regular la movilidad en el territorio de su jurisdicción. Este proceso, que implicó el despojamiento de esta facultad a individuos y entidades privadas, constituyó una parte fundamental de la transición del feudalismo al capitalismo, y del proceso de consolidación del moderno sistema de Estados nacionales (Torpey, 2006).

Sin embargo, y a pesar de la temprana intervención estatal en los flujos internacionales de población, "[1]a inmigración fue concebida durante décadas como un fenómeno socioeconómico al que no se le atribuyó significación política" (López, 2005, p. 101). Así lo han puesto de manifiesto distintos especialistas que destacan la falta de atención que ha recibido tradicionalmente el "Estado" en los desarrollos teóricos que, desde fines del siglo XIX, intentan explicar los procesos migratorios internacionales 
(Hollifield, 2000; Massey, 1999; Portes, 1997; Zolberg, 1989; entre otros). Al respecto, Domenech y Pereira sostienen que:

La ausencia formal de la figura del Estado, así como su naturalización, en la producción de conocimiento sobre la migración atraviesa la historia del estudio de la movilidad humana, ligado al devenir histórico de las ciencias sociales, y forma parte del proceso de institucionalización del estudio de la migración en el campo de la investigación social en los distintos contextos nacionales. (2017, p. 84)

A fines de 1970, se configuró un escenario mundial en el que comenzó a generalizarse la problematización de la migración sur-norte como "amenaza" en los países industrializados; a materializarse la faceta más restrictiva de las políticas migratorias en estos últimos -coincidente con el fin de la etapa de "Puertas Abiertas" en Europa-, y al mismo tiempo, a constatarse los escasos resultados que obtenían en su pretensión de detener los flujos migratorios.

Como consecuencia, se asistió a una progresiva politización de las migraciones internacionales a escala global, emergiendo por primera vez como objeto de intervención pública en muchos países, de disputa y controversia en ámbitos parlamentarios y en la arena electoral, como tema de preocupación de la opinión pública, con un protagonismo sin precedentes en los medios de comunicación, e incluso como tópico inevitable de las relaciones internacionales entre países de origen y destino, o de espacios intergubernamentales de mayor alcance (Alba, 1999; Hollifield, 2000; López, 2002, 2005).

Fue en este contexto que el mundo académico comenzó a centrar su interés en la dimensión política de las migraciones internacionales, con una producción científica que empezó a crecer de modo progresivo, mayormente concentrada en instituciones académicas de países del Norte.

El punto de partida lo constituyeron los trabajos pioneros de Aristide Zolberg (1989), advirtiendo que es la organización política del espacio mundial contemporáneo en Estados legalmente soberanos y mutuamente excluyentes, lo que define la especificidad de la migración internacional como un proceso distintivo, y como objeto de reflexión teórica. En la misma línea, Sayad $(1984,2002,2008)$ contribuyó a reconocer que las migraciones constituyen por definición un "hecho político" y que la (in)migración y el Estado nacional deben su existencia el uno al otro, ya que para que exista (in)migración, es necesaria la existencia de los Estados nacionales, esto es, de las fronteras y los territorios nacionales. Al mismo tiempo, el Estado existe en la medida en que es soberano sobre un territorio que, definido nacionalmente, produce la figura del "extranjero".

A partir de aquí y en lo que refiere a la evolución de este campo, Calderón (2006) ha analizado las principales líneas por las que ha atravesado el estudio de la dimensión política de las migraciones internacionales, diferenciando, por un lado, lo que denomina la "Vieja Escuela", concerniente a los estudios que atienden la experiencia política mirada 
desde el país receptor (donde tiene anclaje este artículo) y, por otro, el debate contemporáneo que propone "volver los ojos al país de origen", influido por la corriente del transnacionalismo, que emergió en la década de 1990 consagrando un nuevo paradigma en los estudios migratorios.

Dentro de la Vieja Escuela, se reconocen diversas vertientes de análisis como aquellas más tradicionales dedicadas a estudiar la participación laboral y la organización sindical de la población inmigrante en la sociedad receptora; otras abocadas al estudio del activismo político de los grupos étnicos; al ejercicio electoral y el dilema de la naturalización; y por último y más importante, el campo de las políticas migratorias:

La formulación de políticas migratorias es, tal vez, uno de los procesos que tiene un impacto más directo en la vida de los migrantes. De ahí que investigar las políticas migratorias resulte indispensable como eje de estudio, o como referencia obligada de todo análisis que considere la aproximación metodológica al proceso migratorio desde la perspectiva política. (Calderón, 2006, p. 56)

En efecto, con la incorporación del enfoque político en los estudios migratorios, una de las preocupaciones centrales en los ámbitos académicos ha girado en torno a la interpretación sobre cómo se definen y construyen las políticas hacia la (in)migración. Ahora bien, para entender la producción de conocimiento acumulada en la materia en cualquier espacio geográfico, es útil especificar, siguiendo a López (2005), las tres dimensiones o componentes que las constituyen:

En primer término, están las denominadas políticas de regulación y control, que incluyen todo lo relacionado a las reglas, requisitos y procedimientos que determinan la selección y admisión temporal o definitiva de los "extranjeros", y lo que se denomina control interno. La segunda esfera es la que engloba el conjunto de medidas, prácticas y acciones que regulan la integración ${ }^{1}$ socioeconómica y cultural del inmigrante a la sociedad receptora, que se vincula a las políticas orientadas a proporcionar a los migrantes los servicios derivados de su acceso a derechos sociales y económicos, y que suelen incluir las medidas dirigidas a propiciar el fortalecimiento de las organizaciones étnicas y su participación en las instituciones de la sociedad de acogida. En tercer lugar, está la esfera de la nacionalidad, que si bien no siempre se incluye en los análisis sobre políticas migratorias, de ella depende la transformación en "ciudadanos" de los migrantes y sus descendientes.

1 Según López (2005), el término integración ha adoptado formas muy diversas en la práctica política, la literatura especializada y el discurso público. La confusión que despierta se relaciona, esencialmente, con la multiplicidad de etiquetas con que se ha identificado el término, asociado a modelos como el de asimilación, melting pot, las estrategias de convivencia cultural, así como con enfoques más inclusivos. Más allá de los significados con los que aparece ligado en la literatura, la noción de integración se utiliza para conceptualizar todo lo que sucede después de la llegada de los migrantes internacionales a la comunidad de destino. 
Existe coincidencia en que ha sido en el marco de la dimensión de regulación y control, donde se ha promovido el mayor número de propuestas teóricas que indagan sobre cómo nacen y se transforman las políticas migratorias, la orientación que asumen, así como los criterios de elegibilidad que pueden establecer, además de una infinidad de estudios empíricos sobre el tema (Castles, 2004; Cornelius, Martin y Hollifield, 1994; Meyers, 2000).

Sin embargo, para los fines de este trabajo, vale detenerse en la segunda dimensión referenciada, atinente a las políticas de integración de la población migrante, ya que se ha advertido contemporáneamente que a partir del gradual proceso de descentralización y transferencia de competencias desde los niveles superiores del Estado hacia los inferiores, se observa en algunos contextos nacionales la "configuración de un régimen de gestión pública de la inmigración multinivel" (Gil, 2010, p. 119). En este marco comienza a adquirir centralidad la "escala local" para el análisis de la intervención del Estado en la incorporación de los migrantes en distintas esferas de la sociedad de acogida, a diferencia de los restantes componentes de las políticas públicas migratorias que siguen anclados en la competencia exclusiva y excluyente del Estado central.

A la luz de este breve marco contextual, se recupera seguidamente el recorrido que ha experimentado Argentina en la producción académica sobre el fenómeno migratorio y el papel del Estado a través de la elaboración de políticas en la materia, para plasmar la desconsideración histórica del rol que desempeñan los gobiernos locales en este campo.

\section{La evolución de los estudios migratorios en Argentina y el abordaje de la producción de conocimiento sobre las políticas migratorias ${ }^{2}$}

En Argentina, la inmigración internacional constituye uno de los procesos más relevantes de la historia nacional, debido a la influencia que ejerció en la configuración económica, política, social y cultural de esta nación desde los inicios de su etapa independiente, sumado a su impacto demográfico que no tuvo correlato a nivel latinoamericano. En efecto, entre 1857 y 1914, este país recibió 4.600.000 inmigrantes, en su mayoría europeos, y para este último año, el $29,9 \%$ de los habitantes del país eran extranjeros (Devoto, 2003). Luego de la segunda mitad del siglo XX, el valor proporcional de la inmigración fue descendiendo y no logró nunca superar los niveles mencionados. Por su parte, la inmigración limítrofe, y luego, regional, comenzó desde entonces a incrementar

2 Se ha realizado un esfuerzo de síntesis para ajustar el trabajo a las normas de extensión de esta revista, dejando de lado distintas referencias bibliográficas. Entre ellas, por ejemplo, toda la producción asociada a las políticas de refugio que están directamente asociadas a las políticas migratorias (sobre lo cual se recomienda consultar investigaciones de Stephanie Mc Callum, María Paula Cicogna, Janneth Clavijo, y otros); o dimensiones más específicas que atraviesan los estudios de políticas migratorias, como la perspectiva de género, siendo en este caso las contribuciones pioneras de María José Magliano, las que inauguran este debate en el país. 
su peso relativo en relación con la europea, alcanzando en la actualidad a más del $80 \%$ del total de la población migrante de este país (Instituto Nacional de Estadísticas y Censos [INDEC], 2010), que supera en total los dos millones de personas según las últimas proyecciones.

Debido a esta razón, los primeros antecedentes del abordaje académico de la temática migratoria en Argentina pueden ubicarse a fines del siglo XIX, en el contexto del desarrollo de la inmigración masiva de ultramar (1880-1914), aunque adquiriendo un carácter más sistemático desde mediados del siglo XX, con la influencia indiscutida de los trabajos de Gino Germani. Este sociólogo, precursor y referente de la sociología argentina y latinoamericana, "pivoteando sobre la idea de integración, destaca el rol de la inmigración europea en el desarrollo y modernización de la sociedad nacional " (Pacecca y Courtis, 2008, p. 15), dejando una huella irrefutable en el debate sociológico e historiográfico sobre la migración.

Hay una característica no menor que destacan Domenech y Pereira (2017) respecto del desarrollo e institucionalización de los estudios migratorios en el país, y es que, a diferencia de lo sucedido en otros ámbitos nacionales, en Argentina no se desarrolló una especificidad disciplinar en la investigación sobre el tema, sino, por el contrario, la construcción de un campo científico multidisciplinar, reconocido como el de los "estudios migratorios", con aportes procedentes de la demografía, historiografía, sociología y antropología, a la que más tarde se sumaron otras ciencias sociales.

Distintos autores han realizado estados del arte generales o parciales (por disciplina) sobre la producción académica sobre las migraciones en el país (Benencia, 2003; Devoto, 2003; Devoto y Otero, 2003; Domenech y Pereira, 2017; Maffia, 2010; Pacecca y Courtis, 2008; Trpin y Jardim, 2015), siendo la cuarta de las citadas contribuciones una de las más completas y actualizadas con las que se cuenta hasta el presente. Estos aportes permiten reconstruir, transcurridos los antecedentes citados, tres momentos en la consolidación de este campo: una primera generación de investigaciones migratorias, dominada por la disciplina historiográfica, tuvo lugar en las décadas de 1970 y 1980, y se centró ampliamente en el estudio de la inmigración europea, con análisis enfocados en las causas del desplazamiento internacional, y también en el papel de las cadenas y redes migratorias, la inserción social en el país (abordando temas como asociacionismo, participación política, movilidad social, pautas matrimoniales, ingreso al mundo del trabajo, entre otros) (Devoto, 2003).

Un segundo momento, desde fines de la década de 1980, pero más concretamente en la subsiguiente, en que la inmigración procedente de países limítrofes y otras procedencias sudamericanas se fue convirtiendo en objeto de reflexión de diversas investigaciones sociales. Si bien constituye esta una inmigración de larga data en Argentina, en los noventa se consolidó como la principal fuente de inmigración del país, inició en un proceso de "hipervisibilidad" étnica (Grimson, 2006), y comenzó a ser objeto de discursos xenófobos 
por parte del aparato estatal y los medios de comunicación, influyendo en la multiplicación de publicaciones sobre el tema.

En este nuevo marco, la producción científica especializada se nutrió, en gran medida, de análisis sociodemográficos abocados al estudio de la relación entre inmigración, empleo y mercados de trabajo; inserción laboral, fecundidad y estructura familiar; distribución espacial y usos del espacio público de los migrantes; procesos identitarios; participación política, etc. Muchos de estos estudios pretendieron someter a evaluación, desde el punto de vista científico, lo que los voceros de las altas esferas del gobierno manifestaban en torno al impacto negativo de los migrantes limítrofes en materia de desocupación, inseguridad, saturación de servicios sociales, entre otros temas (Devoto, 2003; Pacecca y Courtis, 2008). En efecto, esta nueva corriente adquirió especial significancia en el marco de la supremacía que habían tenido hasta aquel momento los estudios sobre la inmigración europea, evidenciando una valoración diferenciada y jerarquizada de ambas corrientes migratorias por parte de las élites académicas.

La última y más importante etapa en la generación de estudios migratorios tuvo lugar a inicios del siglo XXI y llega hasta el presente. Para los fines de este trabajo, vale destacar como rasgo novedoso una injerencia más concreta de disciplinas como el derecho, la ciencia política y las relaciones internacionales, que tuvieron su impacto específico en el estudio de las políticas migratorias (Domenech y Pereira, 2017), logrando esta dimensión un salto cuanti-cualitativo que se detalla más adelante.

Este lapso final volvió mucho más heterogéneo el campo científico, con multiplicidad de enfoques disciplinares, corrientes teóricas y analíticas, estrategias metodológicas, y estudios empíricos centrados notablemente en la inmigración regional, y en menor medida, en la extrarregional (asiática, africana), que dominan contemporáneamente la dinámica inmigratoria del país.

Ahora bien, dado este marco general -que solo tiene pretensiones contextuales-, el interrogante emergente es ¿cómo ha evolucionado específicamente el estudio de las políticas migratorias? Y con este, ¿cuáles son las dimensiones y ejes de análisis que se han priorizado? y ¿cuáles las deudas o asignaturas pendientes?

A saber, a raíz de su condición histórica de Estado receptor, los estudios migratorios en Argentina abarcan una literatura verdaderamente amplia y enfoques de diversas disciplinas, pero el espectro se vuelve mucho más reducido cuando se trata de investigaciones específicamente concentradas en el análisis de las políticas migratorias.

El nacimiento de las investigaciones sobre el tema se ubica en la segunda mitad del decenio de 1980, contexto signado por el retorno de la democracia en el país, que afectó el mundo académico en general, y por la reactivación del rol de la investigación científica en el sistema universitario, en particular. 
En correspondencia con las tres etapas caracterizadas anteriormente, se puede distinguir una primera corriente de producciones, dedicadas al análisis de la formulación de políticas migratorias durante la etapa de la "inmigración temprana" e "inmigración de masas" en Argentina (Devoto, 2003), con predominancia de trabajos de corte historiográfico. Estas publicaciones se detienen en: las imágenes y visiones sobre la inmigración internacional construidas por la élite dirigente que lideró la construcción del Estado Nación y las preferencias de atracción por los grupos europeos; el abordaje de las políticas migratorias desde la prensa escrita; la contradicción existente entre sus facetas normativa y administrativo-burocrática; sumado a producciones que se centran en el desarrollo de políticas migratorias en el proyecto nacional de los gobiernos peronistas, ${ }^{3}$ entre otras (Biernat, 1997, 2007; Devoto, 1989, 2001; Halperin, 1987; Pérez Vichich, 1988; Senkman, 1995; entre otros).

En segundo lugar, se identifica otro conjunto de investigaciones dedicado al estudio de la política migratoria argentina en la etapa contemporánea (últimos decenios del siglo XX hasta el siglo XXI), basados, grosso modo, en el análisis de los instrumentos normativos, programas políticos y discursos oficiales elaborados por los gobiernos nacionales democráticos y de facto (algunos incluyendo la variable del tipo de régimen en el tratamiento político del tema), con específica atención en su impacto en los colectivos migrantes limítrofes y/o procedentes de la región.

En esta nueva camada figuran trabajos elaborados en torno al eje políticas migratorias y discriminación; a la regulación estatal del acceso a la residencia legal y el problema de la irregularidad administrativa -concebido como resultado de prácticas estatales-; o los que se ocupan, dentro de este terreno, del recurso de las amnistías o programas de regularización desarrollados durante esta etapa (Cozzani de Palmada, 2004; Mármora, 1984, 1991; Novick, 2001a y 2001b; Oteiza, Novick y Aruj, 1997; Pacecca, 1997; Torrado, 1991; entre otros).

Pacecca y Courtis (2008) reconocen que en este período aparecen también producciones científicas sobre el acceso de los migrantes a la protección social y otros derechos en el marco de las normativas vigentes, así como los que atienden el modo en que el Estado gestiona la diversidad migratoria, y los diferentes procesos intra e interétnicos, con contribuciones de especialistas como Alejandro Grimson, Elizabeth Jelin, Eduardo Domenech, María Inés Pacecca, entre otros.

Ahora bien, en los primeros años de la década de 2000, se inauguró una nueva corriente de investigaciones que produjo un salto cuanti-cualitativo en la producción científica especializada. Como expone Domenech (2009), esto tuvo una correlación directa con los

3 Hace referencia a los dos primeros gobiernos encabezados por el Gral. Juan D. Perón (1946-1952/19521955), líder político de uno de los movimientos populares más importantes de América Latina en el siglo $\mathrm{XX}$. 
cambios en la política migratoria argentina -a partir de la discusión, debate y aprobación de la Ley de Migraciones $\mathrm{N}^{\mathrm{o}}$ 25.871/2004-, que motivó toda una serie de aportes sobre lo que se denominó la "nueva política migratoria".

Esta tercera fase se inició con una obra compilada por el entonces diputado Rubén Giustiniani (2004), autor de la flamante ley, de la que participaron reconocidos especialistas en el tema, referentes de organismos y organizaciones de la sociedad civil del campo migratorio, que habían formado parte del debate y enriquecido el proyecto para que se convirtiera en ley. Estos y otros trabajos que comenzaron a sumarse, buscaron echar luz sobre aquello que se planteó desde el discurso oficial (también parcialmente adoptado por la academia), como un "cambio de paradigma" de la política migratoria argentina, que había pasado de sustentarse en la doctrina de la seguridad nacional a adoptar un enfoque de derechos humanos, así como a reconstruir el debate social, parlamentario y otros aspectos del proceso que condujeron a su promulgación (Badaró, 2006; Ceriani, 2004; Correa, 2004; Courtis, 2006; Novick, 2004, 2008; Oteiza, 2004; y otros).

Paralelamente, emergieron un conjunto de aportes que, desde una mirada crítica, cuestionaron y marcaron deudas pendientes del nuevo marco legal, contradicciones o componentes heredados que no lograron superarse respecto de los marcos regulatorios previos (Courtis y Pacecca, 2007; Domenech 2009, 2011a y 2011b; Nejamkis, 2012; Nejamkis y Rivero Sierra, 2010; Pacecca y Courtis, 2008).

En este contexto surgieron contribuciones dedicadas a identificar las dificultades en la instrumentación de la nueva norma en espacios territoriales específicos, los cuales trascendieron el ámbito académico, ya que también se elaboraron desde organismos técnicos y la sociedad civil (CEIPIL, 2011; Centro de Estudios Legales y Sociales [CELS], 2005, 2007; CELS y Federación Internacional por los Derechos Humanos [FIDH], 2011; Instituto de Políticas Públicas de Derechos Humanos del Mercosur [IPPDH] y Defensoría del Pueblo de la Provincia de Buenos Aires, 2014; Universidad Nacional de Lanús [UNLA] y Fondo de Naciones Unidas para la Infancia [UNICEF], 2013), mientras otros estuvieron abocados a ámbitos de inserción social migratoria concretos, como el de la educación, la salud, el trabajo, la vivienda, entre otros (Beherán, 2012; Gottero, 2012; Jelin, 2007; Vaccotti, 2012; por mencionar solo algunos casos).

En algunas de estas publicaciones se puso de relieve la brecha existente entre la norma y la práctica de la política migratoria, o los problemas de implementación, y quedó tácitamente planteada la necesaria contemplación del nivel de gobierno local en el ámbito migratorio, aunque sin contar con consideraciones demasiado explícitas sobre su comportamiento. Se evidenciaron, por ejemplo, problemáticas de articulación intraestatal al momento de la garantía efectiva de derechos a la población migrante en distintas esferas públicas; la necesidad de adecuar marcos normativos municipales que entraban en conflicto con la legislación migratoria vigente; o la superposición de agencias con influencia en el campo migratorio en espacios locales, con agendas atomizadas y/o desconectadas. 
Llama la atención también que, durante este último período en la generación de estudios sobre la política migratoria, se profundizó notablemente la escala regional de análisis, con trabajos que se han dedicado a describir y analizar su formulación en el contexto del MERCOSUR y el proyecto de integración regional sudamericano en general. Sin embargo, esto no tuvo una misma evolución en lo que refiere a la consideración del nivel de análisis local en los estudios sobre el tema.

De esta evolución y de las categorías conceptuales antes descritas, puede advertirse que dentro de la literatura producida en Argentina sobre políticas migratorias, la dimensión de gestión y control, ha sido la más profundizada, cuando no la más ponderada en aquellas que ofrecen una mirada integral de la intervención estatal sobre la cuestión migración. En este terreno, se incluye todo lo que tiene que ver con el análisis de las categorías de ingreso y admisión al país establecidas por la normativa migratoria hacia distintos grupos; los criterios y procedimientos que instauran el tipo de residencia al que se accede; los programas y acciones en materia de regularización migratoria; el ámbito del control (fronterizo y de permanencia); el ámbito de detención y expulsión de migrantes, entre otros aspectos. En cambio, las dimensiones de la política migratoria concernientes a la integración socioeconómica y cultural de la población migrante, que afecta la accesibilidad a bienes y servicios públicos en la sociedad de destino, ha sido atendida, pero en menor medida que la esfera anterior. Y más reducida aún ha sido la producción científica sobre la dimensión de las políticas migratorias abocadas a la nacionalidad. ${ }^{4}$

La revisión efectuada revela, asimismo, que la formulación de la política migratoria argentina ha sido interpretada, grosso modo, en el marco de la relación entre la estrategia nacional de desarrollo y las políticas demográficas desde el ámbito normativo del Estado (Novick, 1991, 1997, 2008); a su definición desde ámbitos político-técnicos (Mármora, 1991, 2002, 2003); a su abordaje desde una perspectiva crítica a estos últimos, y el rol de Argentina como parte de organismos internacionales que sostienen un régimen de control global de la migración (Domenech, 2009, 2011a, 2011b); a su entendimiento desde la disciplina de las relaciones internacionales (García, 2017; Nicolao, 2014), o aquellas que ponderan la influencia de las organizaciones de la sociedad civil a nivel nacional y transnacional, como sustento fundamental de su construcción (Badaró, 2006; Correa, 2004; García, 2017). En todos estos esfuerzos se percibe como constante casi invariable la adopción del Estado-nación argentino como unidad de análisis, y son prácticamente inexistentes los aportes que se interrogan sobre el rol que le cabe a los niveles subnacionales del Estado, con unas pocas excepciones que se referencian en el siguiente apartado. ${ }^{5}$

4 Sobresalen contemporáneamente las contribuciones de Courtis y Penchaszadeh (2015) y Penchaszadeh y Courtis (2016).

5 Vale destacar que en los últimos años ha surgido una nueva línea de investigaciones directamente relacionada con los cambios que, desde la asunción del gobierno de Mauricio Macri en Argentina (2015- 


\section{Algunas consideraciones para allanar el camino de investigación sobre elapel de los gobiernos locales en el ámbito migratorio}

Como premisa general, Camós (2017) y Galeote (2017) coinciden en señalar que más allá que las migraciones internacionales cuentan con una dimensión global y transnacional, no debe perderse de vista que los proyectos migratorios que emprenden individuos o grupos familiares se plasman en los ámbitos locales, pues los desplazamientos se producen de una ciudad o localidad concreta dentro de un país hacia otra específica y geográficamente determinada. En efecto, en los entornos urbanos se materializa el "hecho migratorio" en sí mismo, tanto desde el punto de vista de la ciudad emisora como receptora, recibiendo estas un impacto o transformación concreta con formas e intensidades variables.

Esto puede evidenciarse en aspectos tales como el ingreso de los migrantes en segmentos locales del mercado de trabajo (Stefoni, 2009); la convivencia en los barrios, su inserción en escuelas, hospitales, salitas y su acceso a servicios públicos en general; las transformaciones territoriales que supone para algunas comunas el asentamiento económico de los migrantes; o las expresiones culturales que modifican hábitos, prácticas y espacios de la comunidad (Garcés, 2014), por señalar solo algunos aspectos desde el punto de vista del contexto local receptor (Thayer y Durán, 2015).

Más aún, los desafíos contemporáneos que se presentan en el ámbito de la incorporación de los migrantes a la sociedad receptora, refuerzan esta necesidad de poner atención en las dinámicas del ámbito local, y por extensión, al comportamiento de los gobiernos subnacionales:

La inclusión de los migrantes y la implementación de acciones orientadas a garantizar sus derechos obligan a redirigir el análisis hacia las ciudades, municipios y áreas metropolitanas (...)

Pensar las migraciones desde la perspectiva de las ciudades implica trasladar la mirada desde el Estado nacional hacia los Estados locales. Si bien las autoridades locales no suelen participar en la formulación de las políticas migratorias, asumen responsabilidades específicas en relación con las poblaciones migrantes (...) (IPPDH, 2017, pp. 15-16)

En este marco, se ha advertido que:

(...) la gestión local de situaciones planteadas por la migración y el desplazamiento se ha convertido en el principal reto de los entes locales en esta primera parte del Siglo XXI. Simplemente nos hemos convertido en el principal agente de gestión de los procesos de inclusión de la población. (Galeote, 2017, p. 168)

2019), se han impulsado en materia de políticas migratorias, evidenciando su retorno a un paradigma seguritario y de recorte de derechos a las poblaciones migrantes. 
En la misma tesitura, Espinosa (2009) refiere a una tendencia hacia la municipalización del tema migratorio, que indica que el gobierno local está llamado a jugar un papel estratégico, dada su cercanía con la población y su territorio, y su responsabilidad de proveer bienes y servicios públicos de manera adecuada, oportuna y suficiente para satisfacer las necesidades básicas de la población, junto con construir la infraestructura social para que ello suceda. Es decir, representan la institución fundamental en lo que refiere a la dimensión de integración de una política migratoria.

En el mismo sentido, Thayer y Durán (2015) subrayan la importancia del municipio o gobierno local para la política de reconocimiento de derechos a la población migrante en dos sentidos. Primero, en la generación de las políticas necesarias para resguardar los derechos en la primera acogida y para legitimar un reconocimiento en el largo plazo. Segundo, porque la administración local es la institución que está en el mejor pie para transferir hacia los otros niveles del Estado los elementos de conflicto en la incorporación de los migrantes:

Si en el primer sentido el municipio actúa como agente directo en la gestión del reconocimiento de los migrantes, en este segundo sentido participa como una "caja de resonancia" tanto de la realidad migratoria como de las limitaciones de las políticas de reconocimiento que dependen de los otros niveles del Estado. (Thayer y Durán, 2015, p. 137)

De ello se desprende que el desarrollo de una política migratoria requiere tanto de una articulación entre sociedad y Estado, pero también, indefectiblemente, de otra que se genera al interior de este último (Álvarez, 2013). De hecho, hoy en día, el desarrollo de políticas migratorias, dada la multidimensionalidad del fenómeno migratorio, comienza a atravesar distintas esferas de la administración y del entramado institucional (e incluso a convertirse en materia de disputa y conflicto entre distintas carteras del Estado), y por añadidura, a exigir la articulación de sus distintos niveles. Sin ir más lejos, la complementariedad de las competencias ministeriales y municipales respecto de realidades como la discriminación, la vulneración de los derechos laborales, o de los derechos culturales en los establecimientos educativos, vuelve ineludible una coordinación multinivel para abordarlas (Cunill, Fernández y Thezá, 2013).

Domenech y Pereira (2017) señalan que la revisión de los usos de la escala de análisis en los estudios sobre políticas migratorias permitiría modificar y, al mismo tiempo, cuestionar ciertos supuestos implícitos en la manera de comprender su construcción. Estos autores entienden que, si bien algunas investigaciones en Argentina han optado por la escala local, suelen asumir una división u oposición entre lo local y lo nacional que soslaya la complejidad o las ambigüedades inherentes a la construcción del "campo de gobierno de la migración". De esta manera, reconocen que "una perspectiva multiescalar, que muestre las articulaciones entre lo local, lo nacional, lo regional y lo 
global $[\ldots]$ en perspectiva histórica, podría dar lugar a novedosos u originales hallazgos" (Domenech y Pereira, 2017, p. 100).

A raíz de estas consideraciones, vale preguntarse si el nivel de análisis local, en lo concerniente a la relación Estado-migraciones internacionales, abre la puerta a la consideración de aspectos que solo tienen que ver con la instrumentación de las políticas que define el Estado central en materia migratoria, o si también contempla las políticas públicas que los gobiernos subnacionales emprenden en el campo específico.

Al respecto, se ha revelado que, contemporáneamente, en algunos municipios -entre los que se incluye a los latinoamericanos-, se aprecian modificaciones en la configuración de la administración local - de manera reactiva o proactiva- para atender la recepción de migrantes en distintas esferas sociales, más allá de que estas administraciones no cuenten con las competencias formales para afrontar esta situación y, en muchos casos, tampoco con los recursos necesarios (Camós, 2017).

Tomando un caso paradigmático a nivel regional, en 2016 se sancionó la Ley Municipal Nro. 16478/2016, que instituyó la política municipal para la población migrante en la ciudad de San Pablo (Brasil). Si bien la Constitución de este país no otorga competencias en materia de legislación migratoria a los gobiernos estaduales y municipales, se determinó como iniciativa novedosa esta legislación, para cuya aplicación se designó como autoridad a la Secretaría Municipal de Derechos Humanos y Ciudadanía, y entre cuyos propósitos se incluyó:

I - asegurar al inmigrante el acceso a derechos sociales y a los servicios públicos; II - promover el respeto a la diversidad y a la interculturalidad; III - impedir violaciones de derechos; IV - promover la participación social y desarrollar acciones coordinadas con la sociedad civil. (IPPDH, 2017, p. 98).

Se trata de la primera ley dentro del Estado brasileño en instituir directrices para una política pública migratoria en el ámbito municipal (IPPDH, 2017).

Ahora bien, redirigiendo la mirada al caso argentino, también la Constitución Nacional de este país define que es el gobierno federal el encargado de regular la cuestión migratoria ("fomento de la inmigración europea" en los términos anacrónicos en los que se presenta en el artículo 25 de la Carta Magna), siendo atribución del Congreso Nacional proveer lo conducente a la promoción de la misma. No obstante, el artículo 125 dispone que las provincias están habilitadas para celebrar tratados que tengan por fin promover la inmigración; y también tienen la potestad de regular lo concerniente al acceso de la población extranjera a derechos políticos en sus respectivas jurisdicciones. Luego, sus derechos y obligaciones con arreglo al resto de las esferas de la sociedad de acogida, se rigen por la ley nacional de migraciones. 
Al respecto, Nicolao $(2017,2019)$ se ha interrogado sobre el papel que le otorga la norma migratoria vigente (Ley 25.871) a la distribución de competencias entre los distintos niveles del Estado y encuentra que determinados artículos reconocen explícitamente la necesaria participación de las distintas jurisdicciones estatales (nacional, provincial y municipal) para la garantía de derechos a la población migrante, sobre todo aquellas que ingresan en la esfera de integración de la política migratoria, pero sin precisar sus responsabilidades específicas en este esquema. Por su parte, el decreto reglamentario de esta norma (Decreto $N^{\circ}$ 616/2010) no resuelve dicha cuestión, de modo tal que el marco legal carece de un esquema claro de acción y coordinación al interior del aparato estatal.

Como consecuencia de esta situación, algunos trabajos empíricos que podrían identificarse como las primeras aproximaciones al estudio de la construcción de agendas locales en materia migratoria en este país (Cappellini, Carballo, Escobar, Ruiz y Viale, 2011; Nicolao, 2017; Ortiz, 2009), presentan coincidencia en la identificación de: a) problemas de falta de presupuesto para las intervenciones del Estado local que tienen como destinataria a la población migrante; b) ausencia de directrices claras en materia de coordinación interinstitucional entre agencias involucradas en la temática en cada ciudad, que devienen en acciones fragmentarias, abordajes "caso por caso" y derivaciones múltiples de un organismo a otro en la atención de demandas. c) comportamiento predominantemente reactivo de los departamentos municipales, especialmente en intervenciones asociadas a la vulneración de derechos de la población migrante, y por extensión, ausencia de acciones programáticas en la materia; d) una tendencia local de agencias $\mathrm{u}$ organismos que ofrecen una mirada sobre la población migrante que va operando en su despolitización, y en la devaluación de su capacidad de accionar o demandar colectivamente, afectando las posibilidades de acceso y participación de los migrantes en la formulación de políticas públicas locales. En algunos casos, la estructura gubernamental inhibe los reclamos de los migrantes sin rechazarlos o excluirlos, es decir, orientándolos hacia determinadas prácticas (socioculturales, por citar el ejemplo más frecuente): "se los invita a conservar algunas de sus prácticas identitarias a cambio de abandonar reclamos que comprometan la estabilidad o reproducción del sistema" (Cappellini et al., 2011, p. 190). ${ }^{6}$

Con todo, y a raíz de la situación formal previamente caracterizada, se podría anticipar que en la mayoría de los municipios argentinos la cuestión migratoria no cuenta con un lugar preestablecido en la administración local, sino que, en los casos en que es incorporado, lo hace con diversa intensidad, nivel de sostenibilidad en el tiempo, actores impulsores involucrados, carácter predominantemente reactivo de las intervenciones, entre otros aspectos que reclaman profundización a través de futuras investigaciones que amplíen la base empírica.

6 Esta idea ha sido trabajada previamente por Domenech (2007, 2009). 
A su vez, se ha podido constatar que las intervenciones de las administraciones locales en el campo migratorio no siempre presuponen la existencia de unidades o departamentos del organigrama municipal orientados a la población migrante. Por el contrario, generalmente no cuentan con este marco institucional, y el escenario se configura por un conjunto desordenado y atomizado de acciones que pueden emerger de distintas carteras del gobierno local, materializados en programas, proyectos, medidas o prácticas políticas, que tienen a los colectivos migrantes como destinatarios directos, o los incluyen explícitamente dentro de un grupo social más amplio (Nicolao, 2017).

Retomando lo expresado hasta aquí y avanzando en el análisis, se interpreta que la configuración de una agenda de políticas públicas municipales en el ámbito migratorio puede verse influida por diversas variables, que van desde el perfil migratorio del municipio en cuestión hasta las competencias legales de las jurisdicciones locales en la legislación migratoria, pasando por otros aspectos que permean la formación de cualquier agenda pública, como el sentido de oportunidad política para el tratamiento del tema en un determinado contexto histórico (Koopmans y Statham, 2000), la existencia de "actores sociales impulsores" o "agenda setters", con capacidad para inscribir el tema en la agenda pública local, o la existencia de "mecanismos disparadores" o "issues mediáticos" (Cappellini et al., 2011), que involucren a la población migrante, logrando una reacción o respuesta por parte del gobierno municipal de turno.

Claro está que otras variables atravesarán ineludiblemente abordajes de esta naturaleza: la escala de los "gobiernos locales" (no todos los gobiernos locales pueden ser considerados iguales $\mathrm{y}$, por ende, sus burocracias tampoco lo son en cuanto a su capacidad organizacional o técnico-administrativa, presupuestaria, nivel de profesionalización, etc.), o la dimensión espacial (región o área geográfica en la que cada municipio se emplaza dentro del mapa argentino).

Por último, todo análisis que pretenda allanar esta línea de investigación no debe perder de vista el proceso descentralizador (definido por la transferencia y desconcentración de actividades públicas desde el Estado nacional hacia las esferas subnacionales) que emprendió Argentina desde la década de 1990, el cual condujo a los municipios a iniciar un proceso de redefinición de sus roles y estrategias de intervención en su territorio, muchas veces sin contar con las herramientas y capacidades institucionales y de gestión necesarias. A partir de este proceso se agregaron a sus funciones tradicionales (obra pública, provisión de servicios básicos, regulación de la vida comunitaria) nuevas atribuciones en materia de seguridad pública, educación, empleo, desarrollo económico local, salud, justicia, entre otros (Iturburu, 2007), que afectan su posicionamiento, herramientas y responsabilidades frente a la inserción social de la población migrante. 


\section{Conclusiones}

La lenta incorporación del enfoque político en los estudios migratorios, el mayor interés relativo brindado por la academia a la esfera de regulación y control por sobre el de integración en los análisis de políticas migratorias, la tardía injerencia de disciplinas vinculadas al estudio del Estado y la administración pública en la evolución de este campo científico, entre otras razones, han contribuido a que los círculos académicos en Argentina no se hayan preocupado por atender el desafío que afrontan los gobiernos locales en el diseño y/o implementación de políticas hacia la población migrante.

Este artículo ha planteado que si bien hay dimensiones de la política migratoria que siguen ancladas en la competencia exclusiva y excluyente del Estado central, existen otras como la esfera de la integración, que ya no pueden pensarse sino a través de una óptica de actuación estatal multinivel, siendo el gobierno local la administración que afronta el reto inmediato en la incorporación de la población migrante a la sociedad receptora.

Fue a inicios del siglo XXI que los cambios acaecidos con la promulgación de la ley 25.871 y la adopción de una política migratoria con enfoque de derechos humanos promovieron en Argentina el inicio de investigaciones centradas en la implementación de este marco legal en territorios específicos, potenciando la escala local de análisis en las investigaciones sobre el tema. Sin embargo, más allá de estas aproximaciones, el papel desempeñado por las unidades de gobierno locales en el ámbito migratorio retiene aún estatus de área de vacancia en este campo de estudios.

El desafío de allanar este camino responde, grosso modo, a que hoy en día se hace evidente que como consecuencia de procesos descentralizadores, los municipios argentinos son los responsables primarios de garantizar el acceso de sus poblaciones -incluidos los grupos migrantes- a un conjunto de bienes y servicios sociales de manera suficiente y pertinente. Además, en el ámbito estricto de las políticas migratorias, deben establecer los mecanismos institucionales y esquemas de articulación intragubernamental que faciliten la implementación de la legislación migratoria vigente en todas sus facetas y aristas. Pero sumado a este rol instrumentador, la particular dinámica que puede asumir la presencia migrante en cada territorio, hace que los municipios estén expuestos a la recepción de demandas variadas y dinámicas en cuanto al complejo proceso de inserción social de los migrantes, y por extensión, su comportamiento puede asumir un espectro amplio de manifestaciones, que solo puede comprenderse en profundidad, promoviendo líneas de investigación sobre el tema.

Esta necesidad, además, se fortalece a la luz del patrón de distribución de la población migrante en Argentina, que revela una preferencia en ascenso por su radicación en grandes centros urbanos, capitales de provincia, y crecientemente, ciudades intermedias. Así, mientras el porcentaje de población no nativa sobre la población total a escala nacional es 
de 4,5\%, en algunas ciudades del país alcanza hasta el $15 \%$ de los habitantes (INDEC, 2010), dinámica receptiva que amplifica el desafío planteado.

Este trabajo buscó aportar, a través de la revisión bibliográfica, una reflexión sobre el papel de los municipios en el ámbito migratorio, por considerarlo uno de los retos más importante en la gestión pública de las migraciones en la actualidad en un país de tradición receptora como Argentina, que no ha recibido la atención suficiente del campo académico. En tal sentido, se asume que disciplinas como la ciencia política y otras afines al estudio del Estado y las políticas públicas están llamadas a realizar importantes contribuciones en este campo.

\section{Referencias bibliográficas}

Alba, F. (1999). La política migratoria mexicana después de IRCA. Estudios Demográficos y Urbanos, 1(14), 11-37.

Álvarez Enríquez, L. (2013). Interculturalidad: inclusión y exclusión en la política de gestión de la diversidad en Barcelona. Revista del CLAD Reforma y Democracia, 57, 123-156.

Badaró, M. (2006). La conciencia y la ley: la cuestión migratoria en las prácticas de agencias estatales y organismos no gubernamentales en la ciudad de Buenos Aires. En A. Grimson y E. Jelin (comps.), Migraciones regionales hacia la Argentina: diferencia, desigualdad y derechos (pp. 207-235). Buenos Aires: Prometeo.

Beherán, M. (2012). Migraciones y educación en Argentina. Transformaciones y continuidades. En S. Novick (ed.), Migración y políticas públicas. Nuevos escenarios y desafios (pp. 201-226). Buenos Aires: Catálogos.

Benencia, R. (2003). Apéndice. La inmigración limítrofe. En F. Devoto (ed.), Historia de la inmigración en la Argentina (pp. 433-484). Buenos Aires: Sudamericana.

Bertoni, L. A. (1992). La naturalización de los extranjeros, 1887-1893: ¿Derechos políticos o nacionalidad? Desarrollo Económico, 32(125), 57-77.

Biernat, C. (1997). Prensa diaria y política migratoria del primer peronismo: dificultades y aciertos en la construcción de una opinión pública (1945-1955). Estudios Migratorios Latinoamericanos, 14(43), 277-302.

Biernat, C. (2007). ¿Buenos o útiles? La política inmigratoria del peronismo. Buenos Aires: Biblos.

Calderón Chelius, L. (2006). El estudio de la dimensión política dentro del proceso migratorio. Sociológica, 21(60), 43-74.

Camós, I. (2017). La participación de los gobiernos locales en las políticas de inmigración: las competencias municipales en materia de inmigración. Inmigración y gobierno 
local. Experiencias y retos. IV Seminario Inmigración y Europa. [Documento del CIDOB en línea]. Recuperado de

https://www.cidob.org/es/media2/publicacions/monografias/iv_seminari_migracion s/03_camos

Cappellini, N., Carballo, C., Escobar, M., Ruiz, A. y Viale, G. (2011). Conformación de la agenda gubernamental y alcance de las prácticas políticas: el caso de los migrantes bolivianos en Córdoba. En C. Pizarro (ed.), «Ser boliviano» en la región metropolitana de la ciudad de Córdoba. Localización socioespacial, mercado de trabajo y relaciones interculturales (pp. 165-192). Córdoba: EDDUC.

Castles, S. (2004). Why migration policies fail? Ethnic and Racial Studies, 27(2), 205-227.

Centro de Estudios Legales y Sociales (CELS) (2005). A dos años de nueva Ley de Migraciones: avances, cuestiones pendientes y puntos preocupantes. En CELS (comp.), Derechos Humanos en Argentina. Informe 2005 (pp. 337-360). Buenos Aires: Siglo XXI.

CELS (2007). Derechos de las personas migrantes luego de la sanción de la nueva Ley de Migraciones 25.871: sin cambios efectivos. En CELS (comp.), Derechos Humanos en Argentina. Informe 2007 (pp. 293-309). Buenos Aires: Siglo XXI.

CELS-Federación Internacional por los Derechos Humanos (FIDH) (2011). Argentina. Avances y asignaturas pendientes en la consolidación de una política migratoria basada en los derechos humanos. [PDF en línea]. Recuperado de http://www.cels.org.ar/common/documentos/CELS.FIDH.Migrantes.pdf

CEIPIL (2011). Las organizaciones de inmigrantes latinoamericanos en el interior de la provincia de Buenos Aires y el acceso a derechos sociales. Tandil y Pinamar como estudios de caso. Informe elevado al Observatorio Social Legislativo de la Honorable Cámara de Diputados de la Provincia de Buenos Aires. Tandil: UNCPBA.

Ceriani, P. (2004). Nueva Ley: un paso hacia una concepción distinta de la migración. En R. Giustiniani (Comp.), Migración: un derecho humano (pp. 113-135). Buenos Aires: Prometeo.

Cornelius, W. A., Martin, P. L. y Hollifield, J. (1994). Controlling Immigration: A Global Perspective. Stanford CA: Stanford University Press.

Correa, V. (2004). La nueva ley de migraciones y la participación de las organizaciones de la sociedad civil. En R. Giustiniani (comp.), Migración: un derecho humano (pp. 175-179). Buenos Aires: Prometeo.

Courtis, C. (2006). Hacia la derogación de la Ley Videla: la migración como tema de labor parlamentaria en la Argentina de la década de 1990. En A. Grimson, y E. Jelin (comps.), Migraciones regionales hacia la Argentina. Diferencia, desigualdad y derechos (pp. 162-205). Buenos Aires: Prometeo.

Courtis, C. y Pacecca, M. I. (2007). Migración y derechos humanos: una aproximación crítica al "nuevo paradigma" para el tratamiento de la cuestión migratoria en 
Argentina. Revista Jurídica de Buenos Aires, Número Especial sobre Derechos Humanos, 183-200.

Courtis, C. y Penchaszadeh, A. P. (2015). El (im)posible ciudadano extranjero. Ciudadanía y nacionalidad en Argentina. Revista SAAP, 9(2), 375-394.

Cozzani de Palmada, M. R. (noviembre, 2004). Inmigrantes limítrofes en Argentina. ¿Tolerancia o rechazo? Amérique Latine Histoire et Mémoire. Les Cahiers ALHIM. [En línea]. Recuperado de http://alhim.revues.org/document37.html

Cunill Grau, N., Fernández, M. y Thezá Manríquez, M. (2013). La cuestión de la colaboración intersectorial y la integralidad de las políticas sociales: lecciones derivadas del caso del sistema de protección a la infancia en Chile. Polis Revista Latinoamericana, 12(36), 289-314.

Devoto, F. (1989). Políticas migratorias argentinas y flujo de población europea. Estudios Migratorios Latinoamericanos, 11, 135-158.

Devoto, F. (2001). El revés de la trama: políticas migratorias y prácticas administrativas en la Argentina (1919-1949). Desarrollo Económico, 41(162), 281-304.

Devoto, F. (2003). Historia de la inmigración en la Argentina. Buenos Aires: Sudamericana.

Devoto, F. y Otero, H. (2003). Veinte años después. Una lectura sobre el crisol de razas, el pluralismo cultural y la historia nacional en la historiografía argentina. Estudios Migratorios Latinoamericanos, 17(50), 181-226.

Domenech, E. (2007). La agenda política sobre migraciones en América del Sur: el caso de la Argentina. Revue Européenne des Migrations Internationales, 23(1), 71-94.

Domenech, E. (2009). La visión estatal sobre las migraciones en la Argentina reciente. De la retórica de la exclusión a la retórica de la inclusión. En E. Domenech (comp.), Migración y política: el Estado interrogado. Procesos actuales en Argentina y Suramérica (pp. 21-69). Córdoba: Universidad Nacional de Córdoba.

Domenech, E. (2011a). Crónica de una 'amenaza' anunciada. Inmigración e 'ilegalidad': visiones de Estado en la Argentina contemporánea”. En B. Feldman Bianco, L. Rivera Sánchez, C. Stefoni y M. Villa (comps.), La construcción social del sujeto migrante en América Latina. Prácticas, representaciones y categorías (pp. 31-77). Quito: FLACSO: Sede Ecuador -CLACSO-Universidad Alberto Hurtado.

Domenech, E. (2011b). La «nueva política migratoria» en Argentina: las paradojas del programa «Patria Grande». En C. Pizarro (comp.), Migraciones internacionales contemporáneas. Estudios para el debate (pp. 119-141). Buenos Aires: Ciccus.

Domenech, E. y Pereira, A. (2017). Estudios migratorios e investigación académica sobre las políticas de migraciones internacionales en Argentina. Íconos Revista de Ciencias Sociales, 58, 83-108.

Espinosa Martínez, S. (2009). Propuesta municipal de atención a migrantes internacionales. En N. Baca Tavira, F. Herrera Tapia y R. González Orihuela 
(coords.), Migración, democracia y desarrollo: la experiencia mexiquense (pp. 139-156). Toluca: Instituto Electoral del Estado de México.

Galeote, I. (2017). La participación de los municipios en las políticas migratorias. Migraciones, gestión sin competencias. En S. Naïr et al. (comps.), Derechos Humanos, migraciones y comunidad local (pp. 164-175). Málaga: FAMSI.

Garcés, A. (2014). Contra el espacio público: criminalización e higienización en la migración peruana en Santiago de Chile. Eure, 40(121), 141-162.

García, M. (2017). Influencia de exiliados e instituciones en el proceso político que liga migraciones y derechos humanos en Argentina (1983-2015). (Tesis de doctorado en Relaciones Internacionales). Recuperada de:

http://rephip.unr.edu.ar/handle/2133/16285

Gil Araujo, S. (2010). Las argucias de la integración: políticas migratorias, construcción nacional y cuestión social. Madrid: Iepala.

Giustiniani, R. (2004). Migración: un derecho humano. Buenos Aires: Prometeo.

Gottero, L. (2012). Políticas de salud, migración y desigualdades: análisis de planificaciones nacionales en epidemiología. En S. Novick (dir.), Migración y políticas públicas. Nuevos escenarios y desafíos (pp. 261-292). Buenos Aires: Catálogos.

Grimson, A. (2006). Nuevas xenofobias, nuevas políticas étnicas en la Argentina. En A. Grimson, y E. Jelin (comps.), Migraciones regionales hacia la Argentina. Diferencia, desigualdad y derechos (pp. 69-99). Buenos Aires: Prometeo.

Halperin Dongui, T. (1987). ¿Para qué la inmigración? Ideología y política inmigratoria y aceleración del proceso modernizador: el caso argentino (1810-1914). En T. Halperin D. (comp.), El espejo de la historia. Problemas argentinos y perspectivas latinoamericanas (pp. 189-238). Buenos Aires: Sudamericana.

Hollifield, J. (2000). The politics of international migration: how can we bring the State back in. En C. Brettell y J. Hollifield (comps.), Migration Theory. Talking Across Disciplines (pp. 137-185). Londres: Routledge.

Instituto de Políticas Públicas de Derechos Humanos del Mercosur (IPPDH) (2017). Migrantes regionales en la ciudad de San Pablo. Derechos politicos y sociales. Buenos Aires: Organización Internacional para las Migraciones-IPPDH.

Instituto de Políticas Públicas de Derechos Humanos del Mercosur y Defensoría del Pueblo de la Provincia de Buenos Aires (2014). Acceso a derechos de las personas migrantes en la provincia de Buenos Aires. Argentina: Instituto de Políticas Públicas de Derechos Humanos del Mercosur y Defensoría del Pueblo de la Provincia de Buenos Aires.

INDEC Argentina (2010). Censo Nacional de Población, Hogares y Vivienda 2010. Recuperado de https:/www.indec.gob.ar/indec/web/Nivel4-CensoNacional-999999-Censo-2010 
Iturburu, M. (2007). Modernización en los municipios argentinos. En D. Cravacuore e I. Ricardo (comps.), Procesos políticos municipales comparados en Argentina $y$ Chile (1990-2005) (pp. 167-209). Buenos Aires: Editorial de la UNQ-Universidad Autónoma de Chile.

Jelin, E. (dir.) (2007). Salud y migración regional. Ciudadanía, discriminación y comunicación intercultural. Buenos Aires: Ides.

Koopmans, R. y Statham, P. (2000). Migration and ethnic relations as a field of political contention: An opportunity structure approach. En: R. Koopmans y P. Statham (comps.), Challenging Immigration and Ethic Relations Politics: Comparative European Perspectives (pp. 13-56). Oxford: Oxford University Press.

López Sala, A. M. (2002). Los retos políticos de la inmigración. Isegoría, 26, 85-105.

López Sala, A. M. (2005). Inmigrantes y Estados: La respuesta política ante la cuestión migratoria. Barcelona: Anthropos.

Maffia, M. (2010). La antropología sociocultural en los estudios migratorios. Un relato exploratorio. Temas de Antropología y Migración, 0, 69-76.

Mármora, L. (1984). Las regularizaciones migratorias y políticas de migración en Argentina. Revista Argentina de Política Económica y Social, 1, 97-109.

Mármora, L. (1991). Migraciones internacionales: la definición de las políticas migratorias. En S. Torrado (comp.), Política y población en la Argentina. Claves para el debate (pp. 189-220). Buenos Aires: Ediciones de La Flor.

Mármora, L. (2002). Las políticas de migraciones internacionales. Buenos Aires: Paidós.

Mármora, L. (2003). Políticas migratorias consensuadas en América Latina. Estudios Migratorios Latinoamericanos, 17(50), 111-141.

Massey, D. (1999). International migration at the down of the Twenty-First Century: the role of the State. Population and Development Review, 25(2), 303-322.

Meyers, E. (2000). Theories of international immigration policy: a comparative analysis. International Migration Review, 34(4), 1.245-1.282.

Novick, S. (1991). Ley y población: la experiencia argentina. En S. Torrado (comp.), Política y población en la Argentina. Claves para el debate (pp.119-174). Buenos Aires: Ediciones de La Flor.

Novick, S. (1997). Políticas migratorias en Argentina. En E. Oteiza, S. Novick y R. Aruj (comps.), Inmigración y discriminación. Políticas y discursos (pp. 83-139). Buenos Aires: GEL.

Novick, S. (2001a). Democracia y población: Argentina 1983-1999. Documentos de Trabajo, 28. Instituto de Investigaciones Gino Germani, Facultad de Ciencias Sociales, Universidad de Buenos Aires. Recuperado de http://lanic.utexas.edu/project/laoap/iigg/dt28.pdf 
Novick, S. (2001b). Un país ¿receptor? Políticas migratorias nacionales. Encrucijadas, 7 , $26-31$.

Novick, S. (2004). Una nueva ley para un nuevo modelo de desarrollo en un contexto de crisis y consenso. En R. Giustiniani (comp.), Migración: un derecho humano (pp. 67-85). Buenos Aires: Prometeo.

Novick, S. (2008). Migración y políticas en Argentina: tres leyes para un país extenso (1876-2004). En S. Novick (comp.), Las migraciones en América Latina. Políticas, culturas y estrategias (pp. 131-171). Buenos Aires: Catálogos-CLACSO.

Nejamkis, L. (2012). Políticas migratorias en tiempos kirchneristas (2003-2010): ¿Un cambio de paradigma? En S. Novick (dir.), Migración y políticas públicas. Nuevos escenarios y desafíos (pp. 89-116). Buenos Aires: Catálogos.

Nejamkis, L. y Rivero Sierra, F. (2010). Patria Grande: consonancias iy disonancias? entre políticas públicas, prácticas políticas y discursos. En S. Novick (dir.), Migraciones y MERCOSUR: una relación inconclusa (pp. 73-94). Buenos Aires: Catálogos y UBA.

Nicolao, J. (2014). La internacionalización de las políticas migratorias. Estudio de caso argentino (2003-2012). (Tesis de doctorado en Ciencia Política). Universidad Nacional de San Martín, Ciudad Autónoma de Buenos Aires, Argentina.

Nicolao, J. (2017). Migraciones regionales y derechos a escala local. Reflexiones en torno al Municipio de General Pueyrredón. Revista de Integración Regional Densidades, $22,113-147$.

Nicolao, J. (2019). Los migrantes regionales en Bahía Blanca (Argentina): desafíos en el acceso a derechos sociales. Estudios Fronterizos, 19(20), 1-25. Recuperado de http://www.scielo.org.mx/pdf/estfro/v20/2395-9134-estfro-20-e024.pdf

Ortiz, C. (2009). Agendas institucionales locales en torno a la nueva Ley de Migraciones: aspectos preliminares de su constitución. El caso de Córdoba. En E. Domenech (comp.), Migración y política: el Estado interrogado (pp. 139-165). Córdoba: UNC.

Oteiza, E., Novick, S. y Aruj, R. (1997). Inmigración y discriminación. Políticas y discursos. Buenos Aires: GEL.

Oteiza, E. (2004). Hacia una nueva política migratoria argentina. Inmigración, integración y derechos humanos. En R. Giustiniani (comp.), Migración: un derecho humano (pp. 87-111). Buenos Aires: Prometeo.

Pacecca, M. I. (1997). Políticas migratorias y administración pública: la Dirección Nacional de Migraciones, 1876-1996. V Congreso Argentino de Antropología Social, Universidad Nacional de la Plata, La Plata, Argentina, julio-agosto. Recuperado de http://www.equiponaya.com.ar/congresos/contenido/laplata/LP4/46.htm

Pacecca, M. I. y Courtis, C. (2008). Inmigración contemporánea en Argentina: dinámicas y políticas. Santiago: CEPAL. 
Penchaszadeh, A. P. y Courtis, C. (2016). Sujetos políticos migrantes y el dilema de la naturalización. ¿Variaciones posnacionales? Colombia Internacional, 88, 159-182.

Pérez Vichich, N. (1988). Las políticas migratorias en la legislación argentina. Estudios Migratorios Latinoamericanos, 10, 441-464.

Portes, A. (1997). Immigration theory for a new century: some problems and opportunities. International Migration Review, 31(4), 799-825.

Sayad, A. (1984). État, nation et immigration: l'ordre national à l'épreuve de l'immigration. Peuples méditerranéens, 27-28, 187-205.

Sayad, A. (2002). Histoire et recherche identitaire, suivi de l'entretien avec Hassan Arfaoui. Saint-Denis: Éditions Bouchène.

Sayad, A. (2008). Estado, nación e inmigración. Revista Apuntes de Investigación del CECYP, 13, 101-116.

Senkman, L. (1995). Perón y la entrada de técnicos alemanes y colaboracionistas con los nazis, 1947-1949: un caso de cadena migratoria. Estudios Migratorios Latinoamericanos, 10(31), 673-704.

Stefoni, C. (2009). Inmigrantes en Chile: una integración diferenciada al mercado laboral. En Fundación Konrad Adenauer Stiftung, Migración y políticas sociales en América Latina (pp. 81-107). Río de Janeiro: Sopla.

Thayer Correa, L. E. y Durán Migliardi, C. (2015). Gobierno local y migrantes frente a frente: nudos críticos y políticas para el reconocimiento. Revista del CLAD Reforma y Democracia, 63, 127-162.

Torpey, J. (2006). Yendo y viniendo. La monopolización estatal de los legítimos "medios de movimiento. Zona Abierta, 116/117, 59-84.

Torrado, S. (1991). Política y población en la Argentina. Claves para el debate. Buenos Aires: Ediciones de La Flor.

Trpin, V. y Jardim, D. (2015). Tendencias de los estudios migratorios en Brasil y Argentina: desafíos actuales. Odisea. Revista de Estudios Migratorios, 2, 134-156.

Universidad Nacional de Lanús (UNLA)-UNICEF (2013). Niñez, migraciones y derechos humanos en Argentina. Estudio a diez años de la ley de migraciones. Buenos Aires: UNLA-UNICEF.

Vaccotti, L. (2012). Inmigración y derecho a la vivienda adecuada en las normas argentinas (1990-2010). En S. Novick (dir.), Migración y políticas públicas. Nuevos escenarios y desafíos (pp. 169-200). Buenos Aires: Catálogos.

Zolberg, A. (1989). The Next Waves: Migration Theory for a Changing World. International Migration Review, 23(3), 403-430. 